\title{
Fully-Integrated Tunable Q-Enhanced Linear Low Noise Amplifier for Wireless Receivers
}

\section{Ziad El-Khatib, Ahmed Al-Gindy, Sherif Moussa}

\begin{abstract}
This paper presents the design of a fully-integrated tunable Q-enhanced LNA resonator filter designed to tune the circuit center frequency and quality factor $Q$. The proposed circuit achieves a $600 \mathrm{MHz} 3 \mathrm{~dB}$ bandwidth tunable center frequency at $2.4 \mathrm{GHz}$ with a $5.5 \mathrm{~dB}$ Quality Factor $Q$ tuning range. The proposed circuit utilize a distortion transistor compensator to improve linearity of the circuit. The results show an $18 \mathrm{dBc}$ of third order intermodulation IM3 cancellation. The overall proposed circuit peak gain is $16.5 \mathrm{~dB}$ and the minimum NF is 0.94 $\mathrm{dB}$ at $2.4 \mathrm{GHz}$ frequency with power consumption of $5.2 \mathrm{~mA}$.
\end{abstract}

Keywords: Low noise amplifier, Tunable Q-enhanced coupled-inductors, Negative resistance and linearization, Wireless communications.

\section{INTRODUCTION}

In practical down conversion radio frequency receivers lossy bulky off-chip filters are used to provide filtering of unwanted out of band interference. However, such off-chip filters degrade the overall noise figure of the system. Using obtain. A technique called Q-enhancement for increasing the $\mathrm{Q}$ of on-chip resonators can be applied to improve the Q factor of the on-chip inductors by placing a negative resistance across the inductors. However, linearity problems exist in these Q-enhanced circuits that degrades the overall dynamic range of the system. The main idea of the proposed circuit is to compensate the loss of coupled-inductors by using active devices transistors to create negative resistance. With this Q-enhanced resonator tuning circuit the resistance of the coupled-inductors is partially cancelled and the effective Q is increased. In this paper, a tunable Q-enhanced LNA resonator filter design circuit architecture is presented based on coupled resonator filter by means of mutual inductance coupled transformer. The proposed tunable Q-enhanced resonator filter designed to tune the quality factor $\mathrm{Q}$ and center frequency and is combined together with a distortion transistor compensator to improve linearity of the circuit. Other published tunable Q-enhanced LNA filter design [1] and [2] and [3] have a high noise figure of over 5

Revised Manuscript Received on February 05, 2020.

* Correspondence Author

Ziad El-Khatib*, Electrical and Computer Engineering, Canadian University Dubai, Dubai, UAE. Email: ziad.elkhatib@cud.ac.ae

Ahmed El-Gindy, department, Electrical and Computer Engineering, Canadian University Dubai, Dubai, UAE. Email: agindy@cud.ac.ae

Sherif Moussa, Electrical and Computer Engineering, Canadian University Dubai, Dubai, UAE. Email: smoussa@cud.ac.ae

(C) The Authors. Published by Blue Eyes Intelligence Engineering and Sciences Publication (BEIESP). This is an open access article under the CC BY-NC-ND license (http://creativecommons.org/licenses/by-nc-nd/4.0/) on-chip inductors with high quality factors $\mathrm{Q}$ are difficult to

dB. And other Q-enhanced LNA filter [1] and [4] and [5] and [6] and [7]. Other published tunable RF LNA Q-enhanced Bandpass filter [8] have degraded linearity of -33 dBm input compression point and $-17 \mathrm{dBm}$ [9] do not employ distortion compensation circuit.

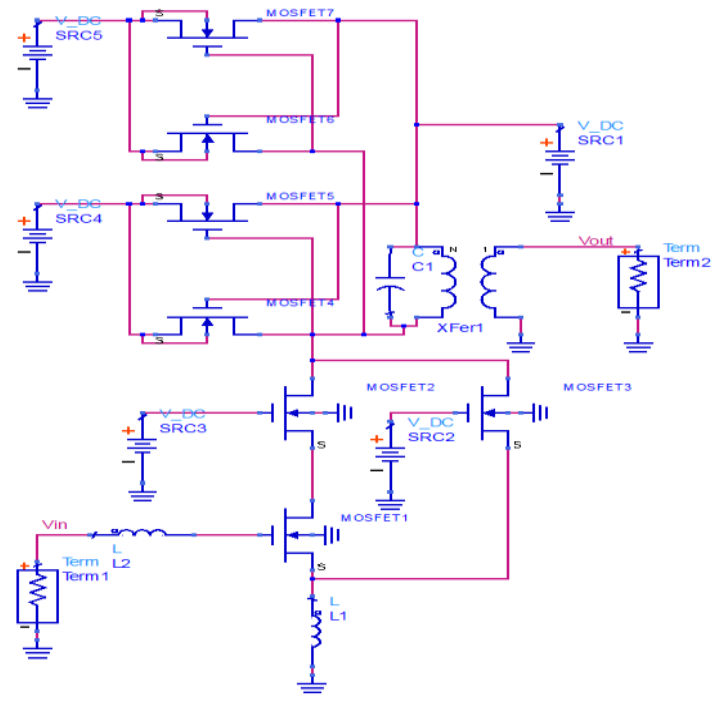

Fig. 1.Proposed fully-integrated tunable Q-enhanced LNA resonator circuit with distortion transistor compensator.

\section{Q-ENHANCED COUPLED RESONANCE FILTER}

\section{A. Q-Enhancement LC resonator tuning circuit}

Resonance LC filter require tuning mechanism to tune the center frequency with quality factor $\mathrm{Q}$ of the circuit. A Q-enhancement tuning mechanism is created from pairs of cross-coupled FET transistors. The main idea is to compensate the loss of inductors by negative resistance. A simple Q-enhanced circuit shown below in Figure 2 where the negative resistance of a cross-coupled pair FET transistors are utilized to partially cancel the parasitic resistance of the on-chip coupled inductor resonator thus enhancing its quality factor $\mathrm{Q}$.
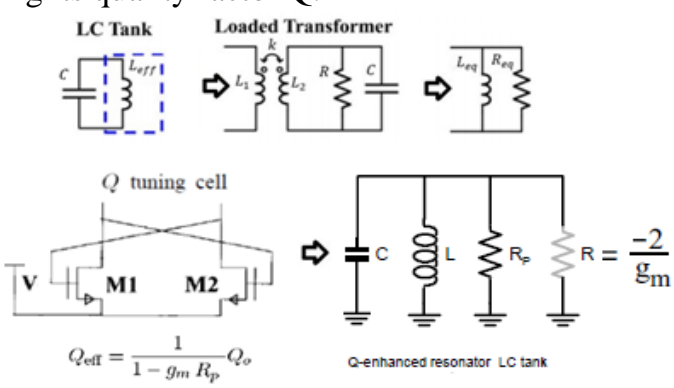

Fig. 2.A basic Q-enhancement $L C$ resonator tuning circuit with cross coupled pair. 
The quality factor $\mathrm{Q}$ is related to $\mathrm{Rp}$ by the following equation. Where transconductance $g_{m}$ is the Q-enhanced MOSFET in cross-coupled pair and Rp is the resistance in parallel with filter

$$
Q=\frac{R_{p} / /\left(-\frac{1}{g m}\right)}{\omega 0 L}
$$

Re-arranging the quality factor $\mathrm{Q}$ above equation we get the following equation

$$
Q=\frac{R_{p} / g m}{\omega_{o} L \cdot\left(\frac{1}{g m}-R_{p}\right)}
$$

The bandwidth BW is related to the center frequency wo and the quality factor $\mathrm{Q}$ by this equation

$$
B W_{3 d B}=\frac{{ }_{0} 0}{\mathrm{Q}}
$$

The center frequency wo is given by

$$
\omega_{o}=\frac{1}{\sqrt{\mathrm{LC}}}
$$

This circuit arrangement partially cancels inductors series resistance and improves boosting $\mathrm{Q}$ factor, where $\mathrm{Q}$ is the parallel resistance $\mathrm{Rp}$ and inductor reactance at resonant frequency by

$$
Q_{0}=\frac{K p}{\omega o L}
$$

The effective $Q_{\text {eff }}$ is adjusted by modifying the cross-coupled pairs transconductance $g_{m}$ of the given by equation [7], [8],

$$
Q_{\mathrm{eff}}=\frac{Q_{o}}{1-g_{m} \mathrm{R}_{v}}
$$

$\mathrm{Q}_{0}$ is the resonant circuit base quality factor.

This the cross-coupled pair circuit behaves as a negative resistance and the amount of negative resistance generated is given by

$$
R=\frac{\mathrm{V}_{d s 1}-\mathrm{V}_{d s 2}}{\mathrm{i}}=\frac{-2}{g_{m}}
$$

The cross-coupled pair negative resistance at high frequencies is given by equation

$$
R=\frac{-2}{g_{m}}\left(1-\frac{1}{g_{m} r_{0}}\right)+\frac{j \omega}{2}\left(C_{g s}+C_{d b}+4 C_{g d}\right)
$$

The filter total capacitance can be regulated tuning the resonance frequency of the resonator filter.

\section{PROPOSED Q-ENHANCED COUPLED-TRANSFORMER TUNING CIRCUIT TECHNIQUE}

The proposed fully-integrated tunable Q-enhanced coupled-transformer resonator circuit CMOS design consists of two pair of cross-coupled transistors connected in parallel configuration where the gates of each device are connected to the drains of the opposite device as shown in Figure 3. The complementary - Gm circuit with supporting bias circuits is a result of using both nMOS and pMOS cross coupled pairs in parallel to generate the total negative resistance tuning mechanism. Hence with proper bias voltage and through suitable setting of the cross-coupled pairs transconductor $\vartheta_{m}$ the effective $Q_{\text {eff }}$ can in theory be tuned as high as desired.

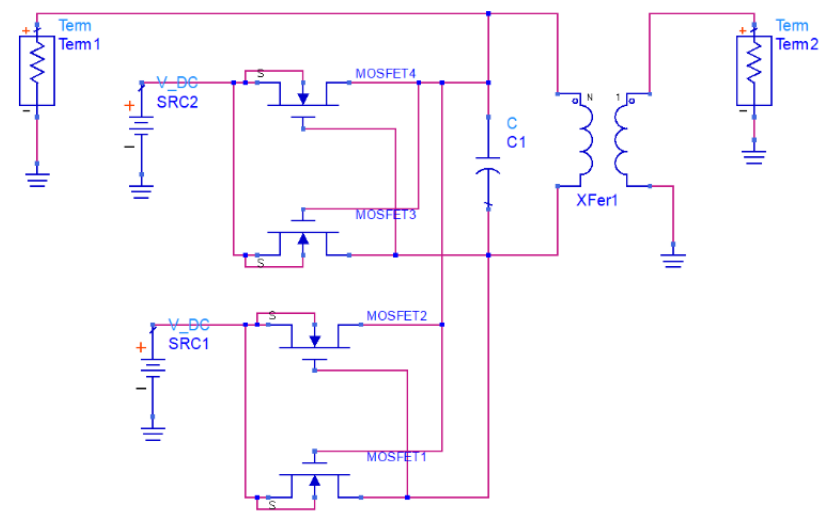

Fig. 3.Proposed Q-enhancement coupled-transformer tuning circuit.

The proposed fully-integrated tunable Q-enhanced coupled-transformer resonator circuit provides a filter $3 \mathrm{~dB}$ bandwidth tuning range of $600 \mathrm{MHz}$ from $2.1 \mathrm{GHz}$ to 2.7 $\mathrm{GHz}$ as shown in center frequency tuning response of the proposed Q-enhanced LNA circuit in Figure 4. The proposed Q-enhanced resonator provides center frequency tuning range and larger 3dB bandwidth then the Q-enhanced LNA filters in [1], [4], [5], [6] and [7].

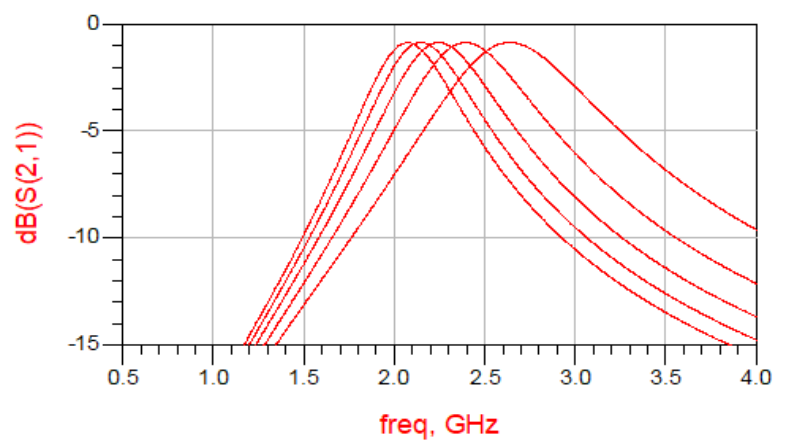

Fig. 4.Center frequency tuning response of the proposed Q-enhanced LNA circuit.

The proposed fully-integrated tunable Q-enhanced LNA coupled resonator filter circuit provides a forward gain S21 of $16.5 \mathrm{~dB}$ and NF of $0.94 \mathrm{~dB}$ at frequency $2.4 \mathrm{GHz}$ as shown in Figure 5. And provides a reflection coefficient of $-30.7 \mathrm{~dB}$.

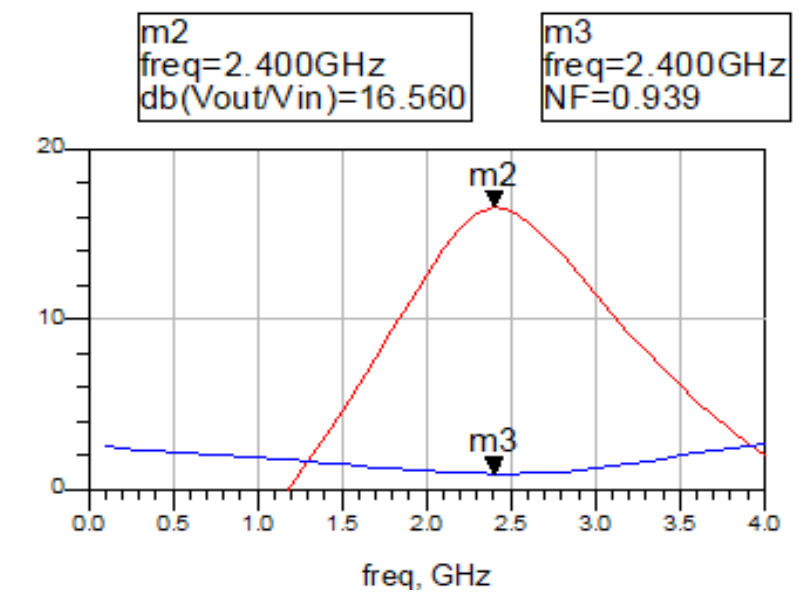

Fig. 5.Response for the proposed Q-enhanced LNA circuit Forward Gain S21 and Noise Figure at frequency $2.4 \mathrm{GHz}$. 
The width of the fully-integrated tunable Q-enhanced LNA design transistors can be determined by the following equation

$$
\mathrm{W}=\frac{1}{3 \cos C_{0 X} R_{g}}
$$

Where $\boldsymbol{w}$ is the operating frequency and $\mathrm{L}$ is the length of the FET transistor and $C_{o x}$ is the transistor oxide capacitance and $R_{g}$ is the source resistance.

$$
R_{s}=\frac{L_{s}}{C} g_{m}
$$

where $L_{g}$ is the source inductance and transistor gate capacitance $\mathrm{C}$ and transconductance $\theta_{\mathrm{m}}$.

The proposed fully-integrated tunable Q-enhanced LNA coupled resonator filter circuit provides a $5.5 \mathrm{~dB}$ quality factor Q tuning range as shown in Figure 6.

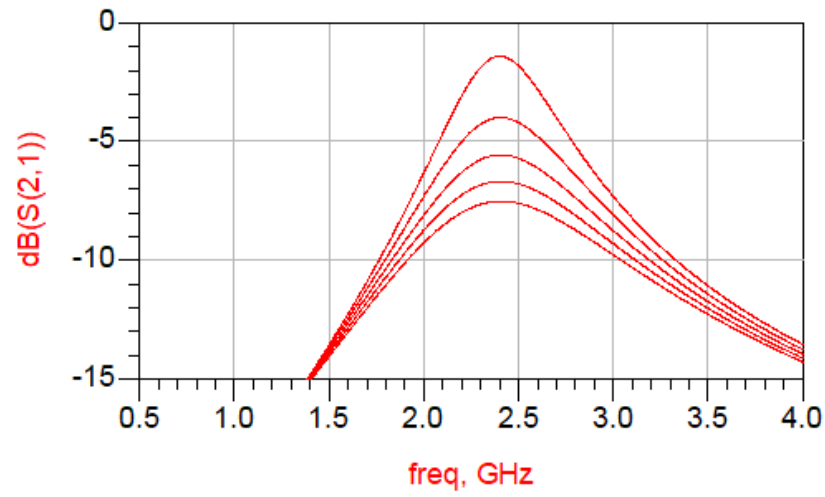

Fig. 6.The Q-enhanced LNA filter $Q$-tuning response at 2.4GHz.

\section{PROPOSED Q-ENHANCED LNA FILTER WITH DISTORTION TRANSISTOR COMPENSATOR}

If The proposed fully-integrated tunable Q-enhanced LNA design utilize a distortion transistor compensator MOSFET3 shown in Figure 1 providing third order intermodulation IM3 distortion cancellation.

The transistor FET drain current is given by Taylor series as [12], [13],

$$
i_{d}=g_{m 1} v_{g s}+g_{m 2} v_{g s}^{2}+g_{m a} v_{g s}^{a}
$$

where $g_{m 1}$ and $\theta_{m 2}$ and $g_{m a}$ are the first transconductance derivative and second transconductance derivative and third transconductance derivative, respectively, with respect to the gate to source voltages.

The third-order intercept amplitude $A_{I I P a}$ is a measure of the circuit nonlinearity and is given by [12], [13],

$$
A_{\text {IIPa }}^{2}=\left|\frac{4 g_{m 1}}{3 g_{m a}}\right|
$$

The LNA circuit third order intercept point amplitude $A_{I I P 3}$ can be written in terms of power and is given by [12], [13],

$$
P_{I I P 3}=20 \log \left(A_{I I P 3}\right)+10 d b m
$$

The LNA circuit $1-\mathrm{dB}$ compression point amplitude $A_{1 d B}$ where $\vartheta_{m 1}$ and $g_{m a}$ the first and third transconductance derivatives is given by [12], [13],

$$
A_{1 d B}^{2}=0.145\left|\frac{g_{m 1}}{g_{m 3}}\right|
$$

The circuit dynamic range DR that can be achieved in a radio frequency receiver with respect to $1 \mathrm{~dB}$ compression point is given by [9], [10],

$$
D R=\frac{P_{1 d B}}{4 K T(F+1) B Q^{2}} Q_{0}^{2}
$$

where is the circuit $\mathrm{P}_{1 \mathrm{~dB}} 1-\mathrm{dB}$ compression point power measured at the LNA's output and $\mathrm{Q}_{0}$ is the base resonant circuit quality factors before and $\mathrm{Q}$ is the quality factor after enhancement. $F_{\text {op }}$ is the circuit operational noise figure and $G$ is the low noise amplifier gain. $\mathrm{B}$ is the bandwidth of the receiver and KT is the Boltzmann's constant times temperature in Kelvin.

With proper bias voltage $\mathrm{vgs}_{\mathrm{gs}}$ and FET transistor size and through suitable setting of the distortion transistor compensator transconductor $\theta_{m}$ the proposed Q-enhanced LNA circuit achieved a third order intermodulation IM3 distortion cancellation of $18 \mathrm{dBc}$ at $2.4 \mathrm{GHz}$ as shown in Figure 7 and Figure 8.

The proposed Q-enhanced LNA simulation response in Figure 7 and Figure 8 show an 18 dBc of IM3 distortion cancellation equivalent of $9 \mathrm{~dB}$ of IIP3 improvement at 2.4 $\mathrm{GH}$ with consumption of $5.2 \mathrm{~mA}$ from a $2.5 \mathrm{~V}$.

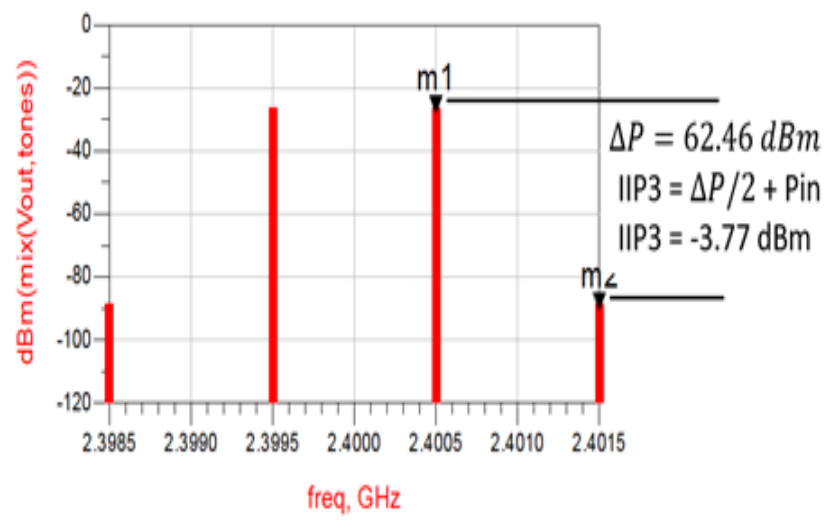

Fig. 7.Response of third-order intermodulation distortion of the proposed Q-enhanced LNA circuit at $2.4 \mathrm{GHz}$

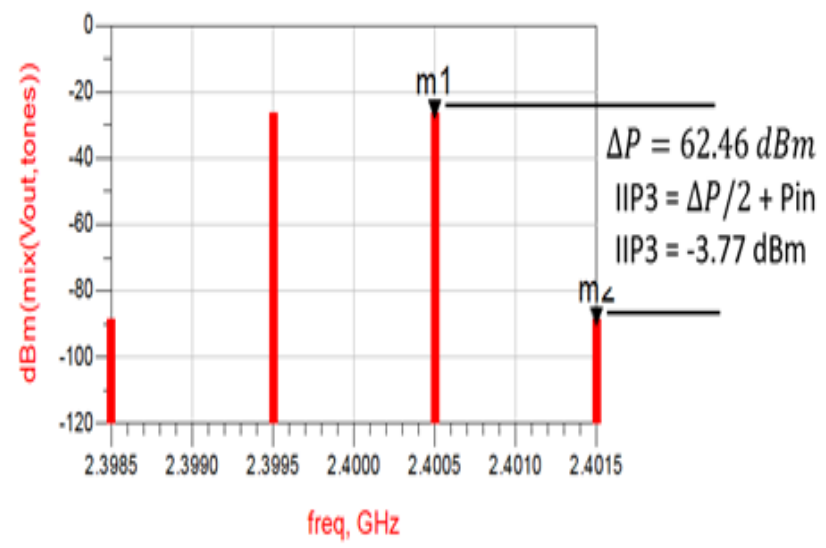

Fig. 8.Response of third-order intermodulation distortion of the proposed Q-enhanced LNA circuit at $2.4 \mathrm{GHz}$ after distortion cancellation.

The IMD3 is improved by $18 \mathrm{dBc}$ and the response of $1-\mathrm{dB}$ Compression point of the proposed Q-enhanced LNA circuit at $2.4 \mathrm{GHz}$ is shown in Figure 9. 
Fully-Integrated Tunable Q-Enhanced Linear Low Noise Amplifier for Wireless Receivers

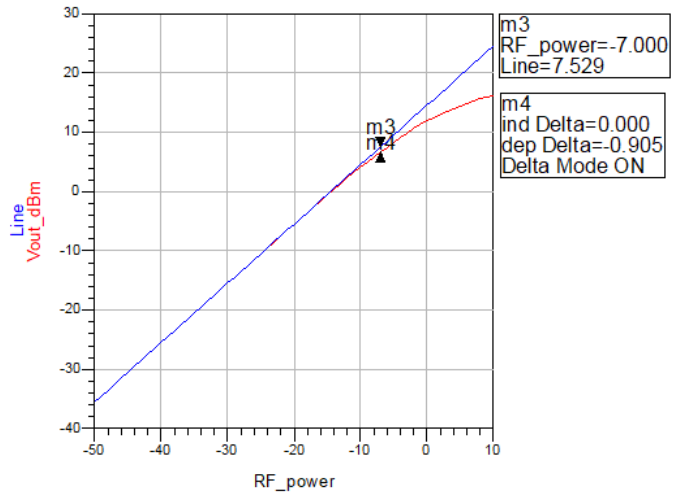

Fig. 9.Response of 1-dB Compression point of the proposed Q-enhanced LNA circuit at $2.4 \mathrm{GHz}$.

\section{PROPOSED Q-ENHANCED LNA TRANSISTOR DIMENSIONS}

The Q-enhanced LNA transistor dimensions are shown in Table I with MOSFET1 and MOSFET2 transistor width size of $100 \mu \mathrm{m}$. The cross-coupled pair transistor width size is $50 \mu \mathrm{m}$. The distortion transistor compensator is $100 \mu \mathrm{m}$. The input matching inductor is $5.7 \mathrm{nH}$ and source degeneration inductor is $1.2 \mathrm{nH}$ shown in Figure 1.

Table- I: Transistor Sizes for the proposed Q-enhanced LNA circuit

\begin{tabular}{|l|l|}
\hline MOSFET1 & $\mathrm{W} / \mathrm{L}: 100 \mu \mathrm{m} / 0.25 \mu \mathrm{m}$ \\
MOSFET2 & \\
MOSFET3 & \\
MOSFET4 & \\
MOSFET5 & $\mathrm{W} / \mathrm{L}: 50 \mu \mathrm{m} / 0.25 \mu \mathrm{m}$ \\
MOSFET6 & \\
MOSFET7 & \\
\hline
\end{tabular}

\section{RESULTS AND DISCUSSION}

The simulation performance results for the proposed tunable Q-enhanced LNA circuit are shown in Table II.

Table- II: Simulation Performance results for the proposed tunable Q-enhanced LNA circuit

\begin{tabular}{|l|l|}
\hline Process & RF CMOS \\
\hline Center Frequency & $2.4 \mathrm{GHz}$ \\
\hline Peak Gain & $16.5 \mathrm{~dB}$ \\
\hline Frequency tuning range & $600 \mathrm{MHz}$ \\
\hline Q-Tuning & $5.5 \mathrm{~dB}$ \\
\hline Noise Figure & $0.94 \mathrm{~dB}$ \\
\hline Reflection Coefficient & $-30.7 \mathrm{~dB}$ \\
\hline Reverse Isolation & $-44 \mathrm{~dB}$ \\
\hline IM3 Tuning & $18 \mathrm{dBc}$ \\
\hline IIP3 Tuning & $9 \mathrm{~dB}$ \\
\hline Power Consumption & $5.2 \mathrm{~mA}$ \\
\hline
\end{tabular}

The comparison of performance of this work and previous Q-enhanced filter in literature is shown in Table III.
Table- III: Comparison between this work and integrated Q-enhanced filter previous work

\begin{tabular}{|c|c|c|c|c|}
\hline REF & $\begin{array}{c}\text { CENTER } \\
\text { FREQ. }\end{array}$ & $\begin{array}{c}\text { 3DB } \\
\text { FREQ } \\
\text { TUNING } \\
\text { RANGE }\end{array}$ & NF & PROCESS \\
\hline$[1]$ & $942.5 \mathrm{MHz}$ & $\begin{array}{c}940 \mathrm{MHz} \\
-982 \mathrm{MHz}\end{array}$ & $\begin{array}{c}6.4 \\
\mathrm{~dB}\end{array}$ & $0.25 \mu \mathrm{m}$ \\
\hline$[3]$ & $1640 \mathrm{MHz}$ & $\begin{array}{c}1500 \mathrm{MHz} \\
-1780\end{array}$ & 2.3 & $0.18 \mu \mathrm{m}$ \\
& & $\mathrm{dB}$ & \\
& & $\begin{array}{c}1.93 \mathrm{GHz} \\
-2.2 \mathrm{GHz}\end{array}$ & 26 & $0.35 \mu \mathrm{dB}$ \\
\hline$[5]$ & $2 \mathrm{GHz}$ & $31.57 \mathrm{MHz}$ & 14.2 & $0.35 \mu \mathrm{m}$ \\
& & -32.76 & $\mathrm{~dB}$ & \\
\hline$[8]$ & $2.4 \mathrm{GHz}$ & $\mathrm{MHz}$ & & \\
& & $2.3 \mathrm{GHz}$ & 15.3 & $0.18 \mu \mathrm{m}$ \\
& & $-2.5 \mathrm{GHz}$ & $\mathrm{dB}$ & \\
\hline$[11]$ & $2.4 \mathrm{GHz}$ & 2.1 & 0.94 & $0.25 \mu \mathrm{m}$ \\
& & $\mathrm{GHz}-2.7$ & $\mathrm{~dB}$ & \\
\hline THIS & $2.4 \mathrm{GHz}$ & $\mathrm{GHz}$ & & \\
WORK & & & & \\
& & & & \\
\hline
\end{tabular}

\section{CONCLUSION}

The design of a fully-integrated tunable Q-enhanced LNA filter circuit design is presented. The proposed circuit achieves a $600 \mathrm{MHz} 3 \mathrm{~dB}$ bandwidth tunable center frequency with a $5.5 \mathrm{~dB}$ Quality Factor Q tuning range. The proposed circuit utilize a distortion transistor compensator to improve linearity of the circuit. The results show $18 \mathrm{dBc}$ of third order intermodulation IM3 cancellation. The overall proposed circuit peak gain is $16.5 \mathrm{~dB}$ and the minimum NF is $0.94 \mathrm{~dB}$ at $2.4 \mathrm{GHz}$ frequency with power consumption of $5.2 \mathrm{~mA}$.

\section{ACKNOWLEDGMENT}

Authors are grateful to all who contributed to the research funding.

\section{REFERENCES}

1. G M. Alkhoury, B. Jarry, "Tunable LNA filter design using coupled-inductor Q-enhancement," Analog integrated circuits and signal processing, 2018.

2. D. Ma, F. Foster Dai, “A 7.27 GHz Q-Enhanced Low Noise Amplifier RFIC With 70 dB Image Rejection Ratio," IEEE Microwave and Wirelesss Components Letters, vol. 20, no. 8, Aug. 2010.

3. S. Wang, R. Wang "A Tunable bandpass filter using Q-Enhanced and Semi-passive Inductors at S-band in $0.18 \mu \mathrm{m}$ CMOS." Progress in Electromagnetics Research, vol. 28, 2011

4. D. Bormann, T. D. Werth, S. Heinen, “A fully integrated Q-enhanced notch filter LNA for TX blocker suppresion in FDD systems," IEEE International Symposium on Radio-Frequency Integration Technology, July 2009.

5. F. Dulger, J. Silva-Martinez, "A 1.3-V 5-mW Fully Integrated Tunable Bandpass Filter at $2.1 \mathrm{GHz}$ in $0.35 \mu \mathrm{m}$ CMOS," IEEE journal of solid-state circuits, vol. 38, 2003.

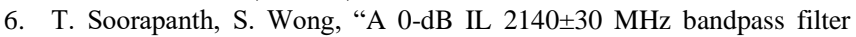
utilizing Q-enhanced spiral inductors in standard CMOS," IEEE journal of solid-state circuits, 2002.

7. W. Kuhn, A. Wyszynski "Q-Enhanced LC Bandpass Filters for Integrated Wireless Applications" IEEE Transactions on Microwave Theory and Techniques, vol. 46, 1998. 
8. A. Hammadi, K. Besbes, "A CMOS $2.4 \mathrm{GHz}$ tunnable RF Bandpass Filter in $0.35 \mu \mathrm{m}$ Technology," International Conference on Design and Technology of Integrated Systems in Nanoscale Era, Jan. 2012.

9. X. He, W. Kuhn, "A $2.5 \mathrm{GHz}$ Low-Power, High Dynamic Range, Self-Tuned Q-Enhanced LC Filter in SOI," IEEE Journal of Solid-State Circuits, vol. 40, no. 8, Aug. 2005.

10. X. He, W. Kuhn, “A Fully Integrated Q-enhanced LC Filter with $6 \mathrm{~dB}$ Noise Figure at $2.5 \mathrm{GHz}$ in SOI," IEEE Radio Frequency Integrated Circuits Symposium, vol. 40, no. 8, Aug. 2004.

11. J. K. Nakaska, J. Haslett, "A CMOS Quality Factor Enhanced Parallel Resonant LC-Tank with Independent $\mathrm{Q}$ and frequency tuning for RF integrated filters," IEEE Transactions on Circuits and Systems-II, July 2005

12. W. Cheng, B. Nauta, "A Wideband IM3 Cancellation Technique using Negative Impedance for LNAs with Cascode Topology," IEEE Radio Frequency Integrated Circuits Symposium, pp: 13, 2012.

13. V. Aparin, "A cellular-Band CDMA 0.25 $\mu$ m CMOS LNA Linearized Using Active Post-Distortion," IEEE Journal of Solid-State Circuits, vo. 41, no. 7, July 2006

\section{AUTHORS PROFILE}

Dr. Ziad El-Khatib $\mathrm{PhD}$ in Electrical and Computer Engineering from Carleton University Canada. Assistant professor at Canadian University Dubai.

Dr. Ahmed Al-Gindy $\mathrm{PhD}$ in Electrical and Communication Engineering from University of Bradford, United Kingdom. Assistant professor at Canadian University Dubai.

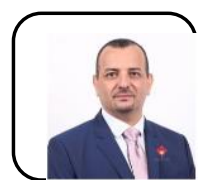

Dr. Sherif Moussa PhD in Electrical and Computer Engineering from University of Quebec Trois-Riviers, Canada. Assistant professor at Canadian University Dubai. 